\title{
Inertia in Temporal Modification
}

\author{
Tim Fernando \\ Trinity College Dublin
}

\section{Introduction}

Inertia is enshrined in Newton's first law of motion, a body at rest or in uniform motion remains in that state unless a force is applied to it. Now, consider (1).

Pat stopped the car before it hit the tree.

Can we conclude from (1) that the car struck the tree? Not without further information such as that supplied in (2).

But the bus behind kept going.

A post-condition for Pat stopping the car is that the car be at rest. To satisfy a pre-condition for the car hitting the tree (namely, that the car not be at rest), inertia requires that some intervening force act on the car (as hinted, for example, by (2)). In the absence of such a force, (1) would appear to suggest that Pat prevented a collision between car and tree.

Exactly what bit of physics are we importing into natural language interpretation here? Oversimplified, Newton's first law of motion says: no change without force. Identifying force with cause, we come to the slogan no temporality without cause, capturing in a phrase the proposal from Steedman 2000 that

temporal semantics of natural language is not primarily to do with time at all. Instead, the formal devices we need are those related to the representation of causality and goal-directed action.

Among the examples Steedman considers is the contrast illustrated in (3).
a. Pat left Dublin but is back (in Dublin).
b. 'Pat has left Dublin but is back (in Dublin).

The acceptability of (3a) brings out, against the oddness of (3b), the need for care if we are to build inertia into natural language interpretation. How can (3a) allow Pat to be back in Dublin when (3b) cannot? An explanation revolving around inertia is offered below. But first, some background.

Appeals to inertia can be traced back in artificial intelligence to the notorious frame problem of McCarthy and Hayes 1969. Within the linguistic semantics literature, references to inertia are made in Dowty 1979 and 1986 for analyzing the progressive and multi-sentential discourse, respectively. These strands of research in AI and linguistics are brought together in Steedman 
2000, and developed further in Hamm and van Lambalgen 2003. The present work is intended as a contribution to this general program, pushing, more specifically, an approach to event semantics outlined in Fernando 2004. Under this approach, "the formal devices" for "the representation of causality and goal-directed action" are finite-state machines: event-types come out as regular languages, and events as strings of observations. Some of these observations are designated below to be inertial, ${ }^{1}$ explaining, in the presence of further assumptions,

(i) why certain uses of before are non-veridical

(ii) how to base temporal for and in modification on conjunctive modification (as with simple Davidsonian cases of event modification)

(iii) why states tend to persist/overlap in a way that events do not

(iv) how a Reichenbachian analysis of the perfect yields existential and resultative readings

(v) incremental change associated, for instance, with the progressive.

\section{Event-types as regular languages}

Let us begin with an example. The regular expression (4) for the un-inflected (tense-less) phrase rain for two days provides strings

$$
\text { rain, } 0(\tau) \text { rain rain, } 2 \text { days }(\tau)
$$

of length $n+2$ (for $n \geq 1$ ), the first symbol in which records rain at time $\tau$, the last symbol, rain 2 days after $\tau$, and the $n$ middle symbols, rain in between.

$$
\text { rain, } 0(\tau) \text { rain }{ }^{+} \text {rain, } 2 \text { days }(\tau)
$$

The formulas rain, $0(\tau)$ and 2 days $(\tau)$ are examples of fluents, assumed to constitute a finite set $\Phi \supseteq\{$ rain, $0(\tau)$, 2days $(\tau)\}$. The alphabet from which regular languages are formed is the power set $\operatorname{Pow}(\Phi)$ consisting of subsets of $\Phi$. The basic intuition is that a string $\alpha_{1} \alpha_{2} \cdots \alpha_{k} \in \operatorname{Pow}(\Phi)^{*}$ describes a temporal sequence of observations, with every fluent in $\alpha_{i}$ asserted to hold at the $i$ th point of the sequence. That is, $\alpha_{1} \alpha_{2} \cdots \alpha_{k}$ amounts to a comic strip or movie beginning with the still picture $\alpha_{1}$, followed by $\alpha_{2}$ and so on, ending with $\alpha_{k}{ }^{2}$ This view is reinforced by adopting the box notation of DRT (Kamp and Reyle 1993), in combination with the notation of regular expressions (in particular, conflating a string $s$ with the singleton language $\{\mathbf{s}\}$ ). Arbitrarily long (but finite) strings are included in (4) to convey the impression of un-interrupted rain over two days (much the same way a movie can give the illusion of continuous motion by flashing a large enough number of stills within a fixed time). 
The longer the string we choose from (4), the more convincing a depiction we have of continuous rain over two days (up to arguably a sufficiently big number, beyond which increasing the length has no discernible effect).

Can we make this Mickey mouse (event-as-comic strip) account more precise? For a model-theoretic interpretation, let us fix a set $\mathrm{Ti}$ of times with a successor relation succ $\subseteq \mathrm{Ti} \times \mathrm{Ti}$, and define a (succ-)run to be a string $t_{1} t_{2} \cdots t_{k} \in \mathrm{Ti}^{*} \operatorname{such}$ that $\operatorname{succ}\left(t_{i}, t_{i+1}\right)$ for $1 \leq i<k$. Now, the idea is that relative to a model $M$ that interprets rain as a subset rain ${ }^{M} \subseteq \mathrm{Ti}$, the string rain, $0(\tau)$ rain rain, 2days $(\tau)$ denotes the set of runs $t_{0} \cdots t_{n+1}$ such that

(i) $\operatorname{rain}^{M}\left(t_{0}\right)$ and $0^{M}\left(\tau^{M}, t_{0}\right)$

(ii) $\operatorname{rain}^{M}\left(t_{i}\right)$ for $1 \leq i \leq n$

(iii) $\operatorname{rain}^{M}\left(t_{n+1}\right)$ and 2 days $^{M}\left(\tau^{M}, t_{n+1}\right)$

with intended interpretations

$$
\begin{array}{rll}
0^{M}\left(t, t^{\prime}\right) & \text { iff } & t=t^{\prime} \\
\text { 2days }^{M}\left(t, t^{\prime}\right) & \text { iff } & t^{\prime} \text { is two days after } t .
\end{array}
$$

(That is, $I(\tau)$ says $I$ time has elapsed since $\tau$, so that in particular, $0(\tau)$ marks the time of the still as $\tau$ ). In general, to interpret a string in $\operatorname{Pow}(\Phi)^{*}$, we must assume that each fluent $\varphi \in \Phi$ can be translated to a formula $\hat{\varphi}[x]$ over a variable $x$. Then, given a model $M$ containing $\mathrm{Ti}$ and interpretations of the translations $\hat{\varphi}[x]$, an $M$-run of a string $\alpha_{1} \cdots \alpha_{k} \in \operatorname{Pow}(\Phi)^{*}$ is a run $t_{1} \cdots t_{k}$ such that

$$
M \models \hat{\varphi}\left[t_{i}\right] \quad \text { for } 1 \leq i \leq k \text { and } \varphi \in \alpha_{i} .
$$

Stepping from a string (event) to a language (event-type), we can accommodate different choices of succ reflecting temporal granularity (the finer the granularity, the longer a run must be of two days).$^{3}$

Models aside, how do we get from rain for two days to (4)? Suppose we were to associate the regular expression (5) with two days.

$$
0(\tau) \square+2 \text { days }(\tau)
$$

To build (5) up to (4), let the superposition $L \& L^{\prime}$ of languages $L, L^{\prime} \subseteq \operatorname{Pow}(\Phi)^{*}$ combine strings from $L$ and $L^{\prime}$ of equal length, forming the componentwise union $\alpha_{i} \cup \alpha_{i}^{\prime}$ of symbols

$$
L \& L^{\prime}=\bigcup_{n \geq 1}\left\{\left(\alpha_{1} \cup \alpha_{1}^{\prime}\right) \cdots\left(\alpha_{n} \cup \alpha_{n}^{\prime}\right) \mid \alpha_{1} \cdots \alpha_{n} \in L \text { and } \alpha_{1}^{\prime} \cdots \alpha_{n}^{\prime} \in L^{\prime}\right\}
$$

Each of $\alpha_{i}$ and $\alpha_{i}^{\prime}$ is understood to be a partial snapshot at some $i$ th time, so that the effect of $\&$ is to overlay motion pictures with the same length. 
$\&$ is a natural form of conjunction (that is, in particular, commutative and associative), mapping regular languages to regular languages (Fernando 2004). We can now construct (4) from (5) as in (6).

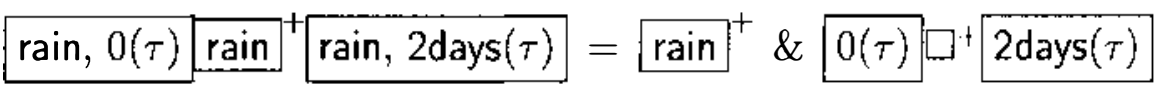

We shall return to (6) below. For now, note that we can apply superposition \& quite freely to combine say, a language $\mathrm{b} / \sim \mathrm{b}{ }^{+}$that marks the initial symbols of its strings (by b) with another language $\sim \mathrm{e}{ }^{+} \mathrm{e}$ that marks the final symbols of its strings (by e).

$$
\begin{array}{|l|l|l|l|}
\hline \mathrm{b} & \sim \mathrm{b} \\
\hline & \sim \mathrm{e} \\
\end{array}
$$

For example, to analyze the phrase Pat eat an apple, let the beginning $\mathrm{b}$ be $\sim(\exists x \sqsubseteq \mathrm{a})$ eat $(\mathrm{p}, x)$ (where $x \sqsubseteq$ a says $x$ is a non-null part of a), and the end e be eat $(p, a){ }^{4}$

Complementing superposition is a form of explicit entailment called subsumption $\unrhd$, defined on languages $L$ and $L^{\prime}$ by

$$
L \unrhd L^{\prime} \quad \text { iff } \quad L \subseteq L \& L^{\prime}
$$

Equating $\mathbf{s} \unrhd \mathbf{s}^{\prime}$ with $\{\mathbf{s}\} \unrhd\left\{\mathbf{s}^{\prime}\right\}$, it follows that over strings,

$$
\alpha_{1} \cdots \alpha_{k} \unrhd \alpha_{1}^{\prime} \cdots \alpha_{n}^{\prime} \quad \text { iff } \quad k=n \text { and } \alpha_{i} \supseteq \alpha_{i}^{\prime} \text { for } 1 \leq i \leq k
$$

while over languages,

$$
L \unrhd L^{\prime} \quad \text { iff } \quad(\forall \mathrm{s} \in L)\left(\exists \mathrm{s}^{\prime} \in L^{\prime}\right) \mathrm{s} \unrhd \mathrm{s}^{\prime} .
$$

Note that (4) $\unrhd(5)$, and that, in general, $L \& L^{\prime} \unrhd L$. Moreover, $\unrhd$ is reflexive and transitive with $\emptyset \unrhd L \unrhd \square^{*}$. And returning to the model-theoretic interpretation of strings above, $L \unrhd L^{\prime}$ implies that every $M$-run of a string in $L$ is an $M$-run of a string in $L^{\prime}$.

\section{Inertia over strings}

Let us call $\varphi$ a post-condition of $L$ if $L \unrhd \square^{*} \varphi$ - that is, every string in $L$ ends with $\varphi$. It is a pre-condition of $L$ if every string in $L$ begins with it: $L \unrhd \varphi \square^{*}$. Returning to sentence (1), let us assume that still-car is a postcondition of the language for the un-inflected phrase Pat stop car, while the negation $\sim$ still-car is a pre-condition of the language for car hit tree. Now, does (1) carry the information that the car hit the tree? A positive answer to this question must square with the fact that the language for the un-inflected phrase (8) must $\unrhd$-subsume (9).

$$
\text { Pat-stop-the-car and-then car-hit-tree. }
$$




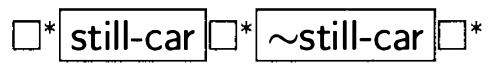

The transition from still-car to still-car in (9) must be accounted for if (1) is to be read as (8). This account may be supplied by the context in which (1) is uttered, or it may even be uttered after (1), as in (2). Otherwise, it is natural to read (1) non-veridically as asserting that the car did not hit the tree.

The argument above assumes that still-car or still-car is inertial, by which we mean that whenever it holds, it persists into the future or back from the past unless some force acts on it. More precisely, let us fix a subset Inr $\subseteq \Phi$ of inertial fluents, and associate with each $\varphi \in \operatorname{Inr}$ a non-inertial fluent $\mathrm{F} \varphi \in \Phi-$ Inr saying: a force is applied on $\varphi$. Bearing this in mind, we may assume (for the sake of simplicity) that $F \varphi$ is the same as $F(\sim \varphi)$. To illustrate with a $\varphi \in \mathrm{Inr}$, inertia turns the string $\square \varphi \square$ to $\varphi \varphi^{\varphi} \mid \varphi$, and $\mathrm{F} \varphi \bar{\varphi} \square$ to \begin{tabular}{|l|l|l|l|l|}
$\mathrm{F} \varphi$ & $\varphi(\varphi)$, but for $\psi \neq \varphi, \mathrm{F} \psi$ & $\varphi \square$ \\
\end{tabular} steps, inertia maps a language $L \subseteq \operatorname{Pow}(\Phi)^{*}$ to the language

$$
\begin{array}{r}
\mathrm{i}(L)=\bigcup_{\mathbf{s} \alpha \alpha^{\prime} \mathbf{s}^{\prime} \in L}\left[\left\{\mathbf{s} \alpha\left(\alpha^{\prime} \cup \varphi\right) \mathbf{s}^{\prime} \mid \varphi \in \alpha \cap \operatorname{lnr}, \mathrm{F} \varphi \notin \alpha\right\} \quad \cup\right. \\
\left.\left\{\mathbf{s}(\alpha \cup \varphi) \alpha^{\prime} \mathbf{s}^{\prime} \mid \varphi \in \alpha^{\prime} \cap \ln , \mathrm{F} \varphi \notin \alpha\right\}\right]
\end{array}
$$

given by the inertial laws (10) and (11).

$$
\begin{aligned}
& \frac{\mathrm{s} \alpha \alpha^{\prime} \mathrm{s}^{\prime}}{\mathrm{s} \alpha\left(\alpha^{\prime} \cup[\varphi) \mathbf{s}^{\prime}\right.} \varphi \in \ln \cap \cap, \mathrm{F} \varphi \notin \alpha \\
& \frac{\mathrm{s} \alpha \alpha^{\prime} \mathbf{s}^{\prime}}{\mathrm{s}(\alpha \cup \varphi) \alpha^{\prime} \mathbf{s}^{\prime}} \varphi \in \ln \cap \alpha^{\prime}, \mathrm{F} \varphi \notin \alpha
\end{aligned}
$$

Notice that $\mathrm{i}(L) \unrhd L$ and that $\mathrm{i}$ distributes over $L: \mathrm{i}(L)=\bigcup_{\mathbf{s} \in L} \mathrm{i}(\{\mathbf{s}\})$.

Let us call a string $\mathbf{s}$ inertially complete (ic) if inertia adds nothing to $\mathbf{s}$ in that $\mathrm{i}(\{\mathbf{s}\}) \subseteq\{\mathbf{s}\}$. (We allow $\mathrm{i}(\{\mathbf{s}\})=\emptyset$ in case every occurrence in $\mathbf{s}$ of an inertial fluent $\varphi$ is accompanied by $F \varphi$.) $\varphi^{n}$ is an example of an ic string; $\varphi \square$ is not (for $\varphi \in \operatorname{lnr}$ ). A language is $i c$ if every string in it is. ${ }^{5}$ For example, $\varphi^{+}$is ic but not $\square^{*} \varphi \square^{*}$ or $\square^{*} \varphi{ }^{*} \square^{*}$. To understand what it takes for a language to be ic, we shall design an operation ic on languages $L$ such that

(a) $i c(L)$ is ic

and

(b) $L$ is ic iff $L=i c(L)$.

Towards that end, let us apply $\mathrm{i}$ repeatedly on $L$, starting with $\mathrm{i}^{0}(L)=L$, moving on for $n \geq 0$ to

$$
i^{n+1}(L)=i\left(i^{n}(L)\right)
$$


and collecting the results in

$$
i^{\omega}(L)=\bigcup_{n \geq 0} i^{n}(L) .
$$

For example, in $\left(\square^{*} \varphi \square^{*}\right)=\square^{*}[]^{n+1} \square^{*}$ and $i^{\omega}\left(\square^{*} \varphi \square^{*}\right)=\square^{*} \varphi^{+} \square^{*}$. Now, the inertial completion of $L$ is the ic fragment of its finitary iterations

$$
i c(L)=\left\{\mathrm{s} \in \mathrm{i}^{\omega}(L) \mid \mathrm{s} \text { is ic }\right\} \text {. }
$$

For example, $i c\left(\square^{*} \varphi \square^{*}\right)=\varphi{ }^{+}$. Clearly, (a) and (b) above hold, and $i c(L)=$ $\bigcup_{s \in L} i c(\{s\}) \unrhd L$. Less obvious perhaps is

Theorem 1. If $L$ is regular then so is ic $(L)$.

The remainder of this section, which the reader may choose to skip, is a proof of this theorem. It is not difficult to see that

$$
i c(L)=i_{\rightarrow} c\left(i_{\leftarrow} c(L)\right)=i_{\leftarrow} c\left(i_{\rightarrow} c(L)\right)
$$

where $i_{\rightarrow}(L)$ is $i c(L)$ with i restricted to the forward rule (10), and similarly for $i_{\leftarrow} c(L)$ and the backward rule (11). Given a finite automaton accepting $L$, with states $Q$, initial state $q_{0}$, final states $F$ and transitions $\rightarrow$, we may form one for $i_{\rightarrow} c(L)$ with

(i) states $Q \times \operatorname{Pow}(\ln r)$

(ii) initial state $\left(q_{0}, \emptyset\right)$

(iii) set $F \times$ Pow(Inr) of final states

(iv) transitions $(q, \gamma) \stackrel{\beta}{\sim}\left(q^{\prime}, \gamma^{\prime}\right)$ iff for some $\alpha$,

$$
q \stackrel{\alpha}{\rightarrow} q^{\prime}, \beta=\alpha \cup \gamma, \text { and } \gamma^{\prime}=\varphi \in \beta \cap \ln \mid \mathrm{F} \varphi \notin \alpha \text {. }
$$

For the inference rule (11), take

(i) states $\left\{q_{0}\right\} \cup(Q \times \operatorname{Pow}(\ln r))$

(ii) initial state $q_{0}$

(iii) set $F \times\{\emptyset\}$ of final states

(iv) transitions

$$
\begin{aligned}
&(q, \gamma) \stackrel{\beta}{\sim}\left(q^{\prime}, \gamma^{\prime}\right) \quad \text { iff } \quad(\exists \alpha) q \stackrel{\alpha}{\rightarrow} q^{\prime}, \gamma^{\prime}=\beta-\alpha \text { and } \\
& \alpha \cup \gamma \subseteq \beta \subseteq \alpha \cup \varphi \in \ln \mid \mathrm{F} \varphi \notin \alpha
\end{aligned}
$$

with no $\leadsto$-transition into $q_{0}$, but

$$
q_{0} \stackrel{\beta}{\sim}\left(q^{\prime}, \gamma^{\prime}\right) \quad \text { iff } \quad(\exists \gamma \subseteq \ln r)\left(q_{0}, \gamma\right) \stackrel{\beta}{\sim}\left(q^{\prime}, \gamma^{\prime}\right)
$$

i.e. $q_{0} \stackrel{\epsilon}{\sim}\left(q_{0}, \gamma\right)$ for $\gamma \in \ln r$. 
Applying these transformations in sequence on an automaton accepting $L$ yields an automaton accepting $i c(L)$.

\section{Regulating inertial flow}

Why are inertial completions interesting? Because they provide a simple way of introducing inertia to natural language interpretation. For the record,

Inertial Hypothesis (IH). The languages interpreting temporal phrases are inertially complete.

Armed with $\mathrm{IH}$, we can derive the analysis (6) of rain for two days from (12) for $\varphi \in \operatorname{lnr}$ and $I$ as in (5), with $0(\tau)$, 2days $(\tau) \notin \operatorname{lnr}$.

$$
\text { for }(\varphi, I)=i c\left(\square^{*} \varphi \square^{*} \& I\right)
$$

Generalizing the language $\varphi^{+}$to which (12) coerces $\square^{*} \varphi \square^{*}$, let us define $L$ to be stative if $i c\left(\square^{*} L \square^{*}\right)=L$. It follows that

$$
L \text { is stative iff } \quad L \text { is ic, } i c\left(\square^{*} L\right) \subseteq L \text { and } i c\left(L \square^{*}\right) \subseteq L \text {. }
$$

The last two conjuncts formalize the persistence of $L$ backwards $\left(\square^{*} L\right)$ and forwards $\left(L \square^{*}\right)$. In addition to $\varphi^{+}$, we have for every $\alpha \subseteq \Phi$, the stative language $\alpha_{\circ}{ }^{*} \alpha\left(\alpha_{\bullet}{ }^{*}\right)$ where $\alpha_{\circ}=\alpha \cap \ln$ consists of all inertial fluents in $\alpha$, and $\alpha$. is the set

$$
\alpha_{\bullet}=\alpha_{0}-\varphi \mid \mathrm{F} \varphi \in \alpha
$$

of fluents in $\alpha_{\circ}$ on which no force is applied in $\alpha$.

But how do we reconcile IH with a transition $\sim \sim \varphi \mid \varphi$ ? There is no denying that for $\varphi \in \ln$,

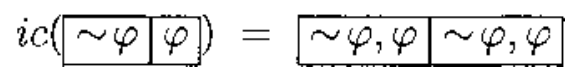

whch we had better avoid if we want only strings with $M$-runs. While $i c$ is $\subseteq$-monotone in that

$$
L \subseteq L^{\prime} \quad \text { implies } \quad i c(L) \subseteq i c\left(L^{\prime}\right)
$$

it is not $\unrhd$-monotone:

$$
L \unrhd L^{\prime} \quad \text { need not imply } i c(L) \unrhd i c\left(L^{\prime}\right)
$$

The side condition " $F \varphi \notin \alpha$ " in (10) and (11) suggests reading $F \varphi$ as "freeze $\varphi$ " (neutralizing inertia). Let us collect F-fluents $F \varphi$ in

$$
\Phi_{\mathrm{F}}=\{\mathrm{F} \varphi \mid \varphi \in \ln r\}
$$


and define an erasure operation $\cdot_{-F}$ that deletes F-fluents from strings

$$
\left(\alpha_{1} \cdots \alpha_{n}\right)_{-\mathrm{F}}=\left(\alpha_{1}-\Phi_{\mathrm{F}}\right) \cdots\left(\alpha_{n}-\Phi_{\mathrm{F}}\right)
$$

and from languages

$$
L_{-\mathrm{F}}=\left\{\mathrm{s}_{-\mathrm{F}} \mid \mathrm{s} \in L\right\} .
$$

Notice that

$$
(\mathrm{F} \varphi, \sim \varphi \mid \varphi)_{-F}=\sim \varphi \mid \varphi
$$

recalling that $\mathrm{F} \varphi=\mathrm{F}(\sim \varphi)$. IH compels us to account for transitions $\sim \varphi \mid \varphi$ by introducing F-fluents that block inertial flow. More precisely, let us say that $L^{\prime}$ accounts for $L$ if

$$
L=\left(i c\left(L \& L^{\prime}\right)\right)_{-\mathrm{F}} .
$$

For example, $\mathrm{F} \varphi \square$ accounts for $\sim \varphi \mid \varphi$. In general, if we set $[\mathrm{F}(L)]$ to apply a force on every inertial fluent occurring in $L$

$$
[\mathrm{F}(L)]=\mathrm{F} \varphi \mid \varphi \in \operatorname{lnr} \text { and }(\exists \mathrm{s} \in L) \mathrm{s} \unrhd \square^{*} \varphi \square^{*},
$$

then $[\mathrm{F}(L)]^{*} \square$ accounts for $L_{-\mathrm{F}}$. We can refine this account as follows. Call $L^{\prime}$ a minimal inertial cover of $L$ if $L^{\prime}$ is ic, $L^{\prime}{ }_{-\mathrm{F}}=L_{-\mathrm{F}}$ and for every $\hat{L}$ such that $(i c(\hat{L}))_{-\mathrm{F}}=L_{-\mathrm{F}}, i c(\hat{L}) \unrhd L^{\prime}$.

Theorem 2. If $L$ is regular, then $L$ has a minimal inertial cover that is regular.

We omit the proof (constructing finite automata), in the interest of space. ${ }^{6}$

Theorem 2 would appear to make IH less an empirical claim and more a methodological assumption. Indeed, minimal inertial cover or not, IH would be vacuous if the effects of inertia were always covered up. Returning to languages $\mathrm{b}, \sim \mathrm{e} \sim \mathrm{b}, \sim \mathrm{e}{ }^{*} \sim \mathrm{b}, \mathrm{e}$ marked by a beginning $\mathrm{b}$ and end $\mathrm{e}$, we can decompose the superposition in (7) as (13), henceforth agreeing that $\varphi^{\mathrm{F}}$ abbreviates ' $\varphi$, F $\varphi$.'

$$
\mathrm{b}, \sim \mathrm{e} \sim \mathrm{b}, \sim \mathrm{e}{ }^{*} \sim \mathrm{b}, \mathrm{e}=i c\left(\overrightarrow{\mathrm{b}^{\mathrm{F}}} \sim \mathrm{b} \square^{*} \quad \& \quad \square *(\sim \mathrm{e})^{\mathrm{F}} \overline{\mathrm{e}}\right)_{-\mathrm{F}}
$$

(13) applies inertia forwards

$$
\mathrm{b} / \sim \mathrm{b}^{+}=i c\left(\mathrm{~b}^{\mathrm{F}} \sim \mathrm{b} \square^{*}\right)_{-\mathrm{F}}
$$

and backwards

$$
\sim \mathrm{e}^{+} \mathrm{e}=i c\left(\square^{*}(\sim \mathrm{e})^{\mathrm{F}} \mathrm{e}\right)_{-\mathrm{F}}
$$

We shall return to (13) in section 6 below.

Turning to temporal in-modification, a first attempt is to adapt (12) to (14) with a transition $(\sim \varphi)^{\mathrm{F}} \varphi$. (For a concrete example, let $\varphi$ be rain, and apply (14) to rain in two days.) 


$$
\operatorname{in}(\varphi, I) \approx i c\left(\square *(\sim \varphi)^{\mathrm{F}} \varphi \square^{*} \& I\right)
$$

Note that

$$
\left.i c\left(\square^{*}(\sim \varphi)^{\mathrm{F}} \varphi \square^{*} \& I\right)=\sim^{4} \sqrt{(\sim \varphi)^{\mathrm{F}}}\right]^{+} \& I
$$

(provided none of the fluents in $I$ are in Inr or $\Phi_{\mathrm{F}}$ ). The backward persistence of $\sim \varphi$ is fine, but the forward persistence of $\varphi$ is arguably not faithful to the meaning of in. There is a telic ${ }^{7}$ requirement in in that we can implement via truncation. More precisely, given $\varphi \in \Phi$, let us call a string $\alpha_{1} \cdots \alpha_{n}$ $\varphi$-truncated if for all $i \in\{1, \ldots n\}$,

$$
\varphi \in \alpha_{i} \text { implies } i=n \text {. }
$$

A language is $\varphi$-truncated if every string in it is. Let $s_{\varphi}$ be the longest prefix of $s$ that is $\varphi$-truncated, and let $L_{\varphi}$ consist of the $\varphi$-truncations of strings in $L$

$$
L_{\varphi}=\left\{\mathbf{s}_{\varphi} \mid \mathbf{s} \in L\right\} .
$$

Clearly, (a) $L_{\varphi}$ is $\varphi$-truncated, (b) $L$ is $\varphi$-truncated iff $L=L_{\varphi}$, and (c) $L_{\varphi}$ is regular if $L$ is. We can now sharpen (14) to (15).

$$
\operatorname{in}(\varphi, I)=i c\left(\square *(\sim \varphi)^{\mathrm{F}} \varphi \square^{*} \& I\right)_{\varphi}
$$

And, for good measure, let us refine the language (5) for two days to (16) $\unrhd(5)$.

$$
0(\tau)<2 \text { days }(\tau)+2 \text { days }(\tau)
$$

It follows that

$$
\operatorname{in}(\varphi,(16))=\sim \sim \varphi{ }^{*}(\sim \varphi)^{\mathrm{F}} \bar{\varphi} \&\left((16)+0(\tau) \mid<2 \operatorname{days}(\tau){ }^{+}\right)
$$

and in general, $\varphi$ in $I$ is read as $\varphi$ in $\leq I$. One of the differences between for $/(12)$ and $i n /(15)$ has to do with durativity. Let us call $L$ durative if $L \unrhd$ $\square \square \square^{+}$- i.e., if all strings in $L$ have length $\geq 3$ (ensuring distinct beginnings, middles and ends). Now, if $I$ is durative, then so too is for $(\varphi, I)$. Not so with in $(\varphi, I)$, thanks to truncation. Of course, it is easy enough to \&-conjoin $\operatorname{in}(\varphi, I)$ with $\square \square \square^{+}$to get a durative language. But that language may well be empty, if all strings in in $(\varphi, I)$ have length $<3$ - a case worth marking out as odd. More in section 6.

\section{Reichenbach's $E, R$ and $S$}

Now for the pair in (3). Assuming for simplicity the un-inflected phrase Pat leave Dublin were given the regular language in $(17),{ }^{8}$ what moral can we draw from (3)? 


$$
\begin{array}{|l|l|}
\hline \operatorname{in}(p, d) & \sim \operatorname{in}(p, d) \\
\hline
\end{array}
$$

A simple analysis of the past tense via a speech time $S$ would associate (18) with PAST(Pat leave Dublin), given (17).

$$
\begin{array}{|l|l|}
\hline \operatorname{in}(\mathrm{p}, \mathrm{d})^{\mathrm{F}} & \sim \operatorname{in}(\mathrm{p}, \mathrm{d}) \\
\hline
\end{array}
$$

Clearly, reducing both Pat left Dublin and Pat has left Dublin to (18) will not yield the contrast in (3). Beyond tense, there is also aspect to consider which we do so, following Reichenbach. ${ }^{9}$

More specifically, aspect falls out of a comparison of a reference time $R$ to an event time $E$ as follows.

Simple. $R=E$

Progressive. $R \sqsubset E \quad(R$ is surrounded by $E)$

Perfect. $R>E \quad(R$ comes after $E)$

In the present setting, let us replace $E$ by a language $L$, and treat $R$ as a non-inertial fluent that marks a position in $L$

$$
\begin{aligned}
\operatorname{Simp}(L, R) & =L \& \square^{*} R \\
\operatorname{Prog}_{(}(L, R) & =L \& \square^{+} R \square^{+} \\
\operatorname{PeRF}_{\circ}(L, R) & =L \square^{*} R .
\end{aligned}
$$

For example,

$$
\begin{aligned}
\operatorname{SimP}\left(\text { rain }^{+}, R\right) & =\text { rain }^{*} \text { rain, } R \\
\text { ProG } \left._{\text {rain }}^{+}, R\right) & =\text { rain }^{+} \text {rain, } R \text { rain } \\
\text { PERF。 } \left._{\text {rain }}^{+}, R\right) & =\text { rain }^{+} \square^{*} R .
\end{aligned}
$$

Were we to think of $R$ as tracking the stage in a computation of $L$, the idea is that

- in the case of $\operatorname{Simp}(L, R), L$ has reached completion

- in the case of $\operatorname{PrOG}(L, R), L$ has not quite gotten there but is on its way

- in the case of $\operatorname{PERF}_{\mathrm{o}}(L, R), L$ is history.

Why the subscript $\circ$ on $\operatorname{PeRF}_{\circ}$ above? If $L$ is ic, so are $\operatorname{Simp}(L, R)$ and $\operatorname{Prog}(L, R)$. But not necessarily $\operatorname{PeRF}_{\circ}(L, R)$. To understand the inertial complications this raises, it is useful to bring in tense, assuming as with $R$ 
that $S \in \Phi-\ln$. Suppose $L$ is a language that an aspectual operator has $R$-marked. The present tense locates $S$ at $R$

$$
\operatorname{PRES}(L, S)=\left(L \quad \& \quad \square * R, S \square^{*}\right) \cap 1(R)
$$

where $1(R)$ consists of all strings in $\operatorname{Pow}(\Phi)^{*}$ in which $R$ occurs exactly once

$$
1(R)=\operatorname{Pow}(\Phi-\{R\})^{*} \operatorname{Pow}(\Phi) \operatorname{Pow}(\Phi-\{R\})^{*} .
$$

The past tense puts $S$ after $R$

$$
\operatorname{PAST}_{\circ}(L, S)=\left(L \square^{*} \& \square^{*} R \square^{*} S \square^{*}\right) \cap 1(R) \cap \text { unpad }
$$

where intersection with unpad truncates leading and trailing $\square$ 's

$$
\text { unpad }=\square+(\operatorname{Pow}(\Phi)-\{\square\})^{+} \operatorname{Pow}(\Phi)(\operatorname{Pow}(\Phi)-\{\square\})^{+} .
$$

As with $\mathrm{PERF}_{\circ}$, we attach a subscript $\circ$ on $\mathrm{PAST}_{\circ}$ to signal unresolved inertial issues.

Returning to Pat left Dublin versus Pat has left Dublin, suppose we were to analyze these as in (19) and (20), respectively.

$$
\begin{aligned}
& \mathrm{PAST}_{\mathrm{o}}(\operatorname{Simp}((17), R), S)=\operatorname{in(\mathrm {p},\mathrm {d})^{\mathrm {F}}\sim \operatorname {in}(\mathrm {p},\mathrm {d}),R\square *S} \\
& \operatorname{Pres}\left(\mathrm{PerF}_{\mathrm{o}}((17), R), S\right)=\operatorname{in}(\mathrm{p}, \mathrm{d})^{\mathrm{F}} \sim \operatorname{in}(\mathrm{p}, \mathrm{d}) \square * R, S
\end{aligned}
$$

Now, the acceptability of (3a) suggests that (19) is fine, which is to say that we do not want inertial flow beyond $R$. By contrast, the oddness of (3b) would be explicable were the post-condition $\sim$ in $(p, d)$ in (17) to flow inertially into $R$, as in the inertial closure (21) of (20).

$$
\begin{array}{|l|l|l|}
\hline \text { in }(\mathrm{p}, \mathrm{d})^{\mathrm{F}} & \sim \operatorname{in}(\mathrm{p}, \mathrm{d}) & \sim \operatorname{in}(\mathrm{p}, \mathrm{d}) \\
\hline
\end{array}
$$

Inertial flow in (21) presents no problem for IH; we simply adopt (22).

$$
\operatorname{PeRF}(L, R)=i c\left(\operatorname{PeRF}_{\circ}(L, R)\right)
$$

The lack of inertial flow in (19), however, needs to be reconciled with IH. With this in mind, let us call $L$ frozen if for all $s \alpha \in L$ and $\varphi \in \alpha \cap \ln r, \mathrm{~F} \varphi \in \alpha$. Next, we define $L^{\bullet}$ so that (a) $L^{\bullet}$ is frozen, and (b) $L$ is frozen iff $L=L^{\bullet}$. This is easy: let $L^{\bullet}=\left\{\mathbf{s}^{\bullet} \mid \mathbf{s} \in L\right\}$ with $\epsilon^{\bullet}=\epsilon$ and

$$
(\mathrm{s} \alpha)^{\bullet}=\mathrm{s}(\alpha \cup \mathrm{F} \varphi \mid \varphi \in \alpha \cap \ln r) \text {. }
$$

(Note that if $L$ is regular, then so is $L^{\bullet}$.) Now, to freeze inertia in (19), let us agree to set

$$
\operatorname{PAST}(L, S)=\operatorname{PAST}_{\circ}\left(L^{\bullet}, S\right)
$$

which is to say: all inertial flow must precede the application of tense. ${ }^{10}$

As it turns out, there are cases where we should form $L^{\bullet}$ before applying (22). Consider the present perfect it has rained. (23) gives two possibilities. 

a. Pres(Perfor $\left.\left(\operatorname{rain}^{-1}, R\right), S\right)=\operatorname{rain}^{+} \square^{*} R, S$
b. Pres(ic(PerFo $\left.\left.\left(\operatorname{rain}^{+}, R\right)\right), S\right)=$ rain $^{\prime}$ rain, $R, S$

If we do not want to infer rain at $S$ from it has rained, we had better opt for (23a), rather than (23b). Indeed, revisiting (3), one may argue that (3b) is acceptable as a reply to the question

\section{Has Pat ever left Dublin?}

It would appear that ever questions license so-called existential readings. Under IH, the difference between an existential and a resultative perfect is whether we freeze $L$ or not (by adding, in the case of $(17), F(\sim$ in $(p, d))$ to the end). An alternative to (22) is (24).

$$
\operatorname{PeRF}(L, R)=\operatorname{PeRF}_{\circ}\left(L^{\bullet}, R\right) \quad[\exists, \text { contra }(22)]
$$

If $L$ is ic, then so is $\operatorname{PERF}_{\circ}\left(L^{\bullet}, R\right)$

$$
\operatorname{PerF}_{\circ}\left(L^{\bullet}, R\right)=i c\left(\operatorname{PerF}_{\circ}\left(L^{\bullet}, R\right)\right)
$$

which is to say we can derive (24) from (22) by first freezing $L$.

\section{Incremental (graded) change}

Two questions about the account of aspect above are

(Q1) How are we to capture the progressive of Pat leave Dublin when

$$
\operatorname{PrOG}(L, R)=\emptyset \quad \text { if } L \text { has no strings of length } \geq 3
$$

as is the case with the language (17) for Pat leave Dublin?

(Q2) Can we account for the widespread view (e.g. Kamp and Reyle 1993) that the progressive and the perfect are stative?

Recall that a language $L$ is durative if all strings in $L$ have length $\geq 3$. Let us call $L \& \square \square \square^{+}$the durative coercion of $L$, noting that (a) $L \& \square \square \square^{+}$is durative, and (b) $L$ is durative iff $L=L \& \square \square \square^{+}$. For similar reasons, let us call $L \& \square \square$ the 2-point coercion of $L$. From (Q1), it is clear that we need to beef (17) up to a language $\hat{L}$ whose (a) 2-point coercion $\unrhd$-subsumes (17) and (b) durative coercion is non-empty. With that in mind, let us collect $L$ 's preconditions in

$$
\alpha_{L}=\varphi \mid L \unrhd \varphi \square^{*}
$$

and $L$ 's post-conditions in

$$
\omega_{L}=\varphi \mid L \unrhd \square^{*} \varphi
$$


and observe that

$$
\begin{aligned}
L \& \square \square & \unrhd \alpha_{L} \omega_{L} \\
L \& \square \square \square^{+} & \unrhd \alpha_{L} \delta_{L}{ }^{+} \omega_{L}
\end{aligned}
$$

where $\delta_{L}$ consists of $L$ 's internal/intermediate invariants

$$
\delta_{L}=\varphi \mid L \& \square \square \square^{+} \unrhd \square \varphi \varphi^{+} \square .
$$

Notice that $\delta_{L}$ is a good candidate for the progressive in (Q2). ${ }^{11}$

Next, recall our analyses (5) and (16) of two days. In both cases, $\alpha_{L}=0(\tau)$ and $\omega_{L}=2$ days $(\tau)$. But $\delta_{L}$ is $\square$ in (5), and $<2$ days $(\tau)$ in (16). To capture the passage of time within the interval, let us introduce a modifier Previously on fluents $\varphi$ that shifts the evaluation time of $\varphi$ one step back

$$
\text { (Previously } \varphi)^{M}(t) \quad \text { iff } \quad\left(\exists t^{\prime}\right) \operatorname{succ}\left(t^{\prime}, t\right) \text { and } \varphi^{M}\left(t^{\prime}\right)
$$

where $M$ is a model containing the successor relation succ on time (interpreting fluents according to section 2). Now, we can refine $\delta_{L}$ to consist of the fluent

$$
(\exists t<2 \text { days }) \text { time }(t, \tau) \wedge\left(\exists t^{\prime}<t\right) \text { Previously time }\left(t^{\prime}, \tau\right)
$$

where time $(2$ days,$\tau)$ and time $(0, \tau)$ re-formulate 2 days $(\tau)$ and $0(\tau)$, respectively. (For control on the increments, we can strengthen $t^{\prime}<t$ to $t^{\prime}<t-\epsilon$, for some parameter $\epsilon>0$.)

Turning to examples where $\delta_{L}$ can be construed as the progressive, let us consider the informal picture below of a durative event $e$.

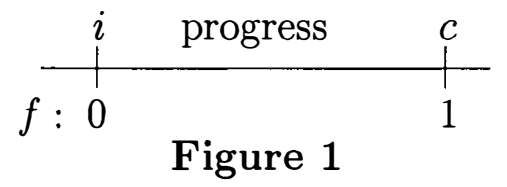

The upper half of Figure 1 follows Moens and Steedman 1988 in representing $e$ by an inceptive event $i$, a culminative event $c$, and a progressive state which is both the consequent state of $i$ and the preparatory state of $c$. The lower half hints at a conceptualization of $e$ in terms of the closed unit interval $[0,1]$ (consisting of real numbers from 0 to 1 ) with the help of a suitable function $f$ that maps $x \in[0,1]$ to a fluent $f(x)$ tracking $e$ up to its $x$ th part (where the $x$ th part of $e$ is $i$ for $x=0$, and all of $e$ for $x=1$ ). Some examples are given in Table 1.

\begin{tabular}{l|l} 
& $f(x)$ for $0 \leq x \leq 1$ \\
\hline Pat swim a mile & 'Pat swim $x \cdot[$ a mile]' \\
Pat grow an inch & 'Pat grow $x \cdot[$ an inch]' \\
Pat drink four pints & 'Pat drink $x \cdot[$ four pints]' \\
Pat drive a car $\underline{50 \mathrm{~km} m s}$ & 'Pat drive a car $x \cdot[50 \mathrm{kms}]$ \\
rain for six hours & 'rain for $x \cdot[$ six hours]'
\end{tabular}

Table 1 
The fluent $f\left(\frac{1}{2}\right)$ corresponds in the first row (Pat swim a mile), to Pat swim half a mile, ${ }^{12}$ and in the last row, to rain for three hours. The items underlined in the left column measure out the event $e$ (in roughly the sense of Tenny 1987), supplying the $x$ th parts that the right column uses to define $f(x)$. (We switch from $e$ to these items because we presumably understand these items better than we do e.)

Recalling line (7) from section 2, we have $f(0)=\mathrm{b}$ and $f(1)=$ e. But what about $f(x)$ for $0<x<1$ ? $^{13}$ Quantifying away that $x$, let $f_{\uparrow}$ be the fluent

$$
(\exists x<1) \quad f(x) \wedge(\exists y<x) \text { Previously } f(y)
$$

that says $e$ is progressing. Accordingly, we can associate regular languages with $i, c$ and progress in Figure 1

$$
\begin{aligned}
\mathcal{L}_{i} & =\mathrm{F} f_{\uparrow}, f(0)^{\mathrm{F}} f_{\uparrow} \\
\mathcal{L}_{c} & =f_{\uparrow}{ }^{\mathrm{F}}, \mathrm{F} f(1) f(1) \\
\mathcal{L} \text { (progress) } & =f_{\uparrow}{ }^{+}
\end{aligned}
$$

where $f(0), f(1)$ and $f_{\uparrow}$ are understood to be inertial. The vertical bars below $i$ and $c$ in Figure 1 mark forces shaping the combination of $\mathcal{L}_{i}, \mathcal{L}_{c}$ and $\mathcal{L}$ (progress) according to Figure 1

$$
\begin{aligned}
& i c\left(\mathcal{L}_{i} \square^{+} \& \square^{+} \mathcal{L}_{c}\right)=i c(\mathcal{L}_{i} \square^{+} \& \square \underbrace{\mathcal{L}(\text { progress })}_{\text {state }} \square \& \square^{+} \mathcal{L}_{c}) \\
& =\begin{array}{|l|l|l|}
\hline \mathrm{F} f_{\uparrow}, f(0)^{\mathrm{F}} & f_{\uparrow} & f_{\uparrow}^{\mathrm{F}}, \mathrm{F} f(1) \\
\hline
\end{array}
\end{aligned}
$$

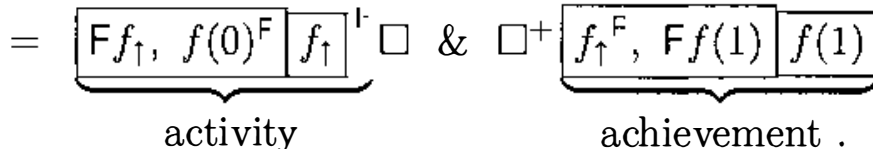

The last line above has the form

activity CAUSE achievement

which accomplishments take in Dowty 1979, with CAUSE reformulated as \&conjunction against an inertial background. The specific case of rain for six hours (the last row of Table 1) suggests sharpening (12) so as not to assert rain at the start or end.

$$
\begin{aligned}
\operatorname{for}(\varphi, I) & =i c\left(\mathrm{~F} \varphi\left(\varphi \square^{*}+\epsilon\right) \varphi^{\mathrm{F}} \square \& I\right) \\
& =\mathrm{F} \varphi \varphi \varphi^{\mathrm{F}} \square \& I
\end{aligned}
$$


(25) treats rain more as an activity than a state - which is not surprising given that the progressive of a state can, as is well-known, be awkward. (25) stays away from the fluent $f_{\uparrow},{ }^{14}$ as we need not restrict ourselves to the $f_{\uparrow^{-}}$ analysis above of Figure 1. For instance, we can associate with Pat bake a cake the language

\begin{tabular}{|l|l|l|l|}
$\operatorname{Fbake}(\mathrm{p}, \mathrm{x}), \sim \operatorname{cake}(\mathrm{x})$ & $\operatorname{bake}(\mathrm{p}, \mathrm{x}), \sim \operatorname{cake}(\mathrm{x})$ & $\operatorname{bake}(\mathrm{p}, \mathrm{x})^{\mathrm{F}}, \sim \operatorname{cake}(\mathrm{x})^{\mathrm{F}}$ & $\operatorname{cake}(\mathrm{x})$ \\
\hline
\end{tabular}

and with Pat walk home

\begin{tabular}{|l|l|l|l|}
\hline Fwalk $(p), \sim \operatorname{home}(p)$ & walk $(p), \sim \operatorname{home}(p)$ \\
\hline walk $(p)^{F}, \sim \operatorname{home}(p)^{F}$ & home $(p)$ \\
\hline
\end{tabular}

(refining both languages, if we wish, by applying $f_{\uparrow}$ to the degree to which, in the former case, $x$ is a cake, and, in the latter, Pat is near home).

Like (17), $\mathcal{L}_{i}$ and $\mathcal{L}_{c}$ have no strings of length $\geq 3$, making ProG $\left(\mathcal{L}_{i}, R\right)$ and PROG $\left(\mathcal{L}_{c}, R\right)$ empty and hence problematic. We are back to (Q1). As with (17), $\mathcal{L}_{i}$ and $\mathcal{L}_{c}$ are 2-point coercions of languages $\mathcal{L}(i)$ and $\mathcal{L}(c)$ from which to form $\operatorname{Prog}(\mathcal{L}(i), R)$ and $\operatorname{PrOG}(\mathcal{L}(c), R)$. Exactly what $\mathcal{L}(i)$ and $\mathcal{L}(c)$ are, I cannot say. Nor do I have a definite answer for the case of (17), which I am inclined to think involves Pat's intentions. That said, I hope this section encourages the reader to explore such questions within the present setting. The trick would be to find the right fluents. With (Q2) in mind, let us mention two well-known binary connectives on fluents that may prove useful: since

$$
\begin{array}{r}
(\varphi \text { since } \psi)^{M}(t) \quad \text { iff } \quad(\exists k \geq 2)\left(\exists \text { run } t_{1} \cdots t_{k}\right) t_{k}=t, \psi^{M}\left(t_{1}\right) \text { and } \\
\varphi^{M}\left(t_{i}\right) \text { for } 1<i \leq k
\end{array}
$$

and until

$$
\begin{array}{r}
(\varphi \text { until } \psi)^{M}(t) \quad \text { iff } \quad(\exists k \geq 2)\left(\exists \text { run } t_{1} \cdots t_{k}\right) t_{1}=t, \psi^{M}\left(t_{k}\right) \text { and } \\
\varphi^{M}\left(t_{i}\right) \text { for } 1 \leq i<k .
\end{array}
$$

These connectives introduce fluents that take snapshots of even more instants than Previously. Indeed, we might try the regular language

$$
\psi \varphi * \varphi, R
$$

before resorting to $\varphi$ since $\psi$ (qua fluent) ${ }_{1}^{15}$ and the reversal

$$
\varphi, R]^{*} \psi
$$

before $\varphi$ until $\psi$. As with anything, since and until must be used with some restraint. In the present context, they suggest candidates for $\delta_{L}$ of the form

$$
\varphi \text { since } \mathrm{b}, \varphi \text { until } \mathrm{e}
$$

from which to construct the progressive state $\delta_{L}{ }^{+}$. For the perfect, we might try a state of the form $\varphi$ since $\psi$, relying on inertia to infer from $\varphi$ that $\varphi$ until Previously $F \varphi$. 


\section{Conclusion}

Let us return briefly to items (i)-(v) ending the Introduction.

(i) To read (1) as (8), we must, under $\mathrm{IH}$, account for the transition from still-car to $\sim$ still-car in (9). More generally, to read $A$ before $B$ as $A$ and then $B$, any conflict between the post-conditions of $A$ and the preconditions of $B$ must be accounted for by an intervening force.

(ii) Against an inertial background, we can base an interpretation of $\varphi$ for $I$ on $\square * \varphi \square^{*} \& I$, subject to boundary conditions ((12) versus (25)) specifying forces around $I$. For $\varphi$ in $I$, we put a force immediately before $\varphi$, alongside $\sim \varphi$, and secure telicity by $\varphi$-truncating $\square^{*}(\sim \varphi)^{\mathrm{F}} \varphi \square^{*}$ before or after superposition with $I$. The latter case yields a reading of in $I$ as in $I$ or less. The former does not (see Endnote 7).

(iii) Call $L$ stative if $L=i c\left(\square^{*} L \square^{*}\right)$.

(iv) Apply (22) - in the case of an existential reading, after freezing $L$.

(v) Form fluents that relate multiple instants (via Previously, since, etc), noting that inertia covers not just rest but also uniform motion (arguably reflecting the progressive).

Among the many questions left open are two which I hope to answer elsewhere: how, within the present approach, to interpret (i) non-veridical readings of before, and (ii) the force construct $\mathrm{F}$ on fluents.

\section{Endnotes}

*My thanks to Cleo Condoravdi and Stefan Kaufmann for helpful discussions.

${ }^{1}$ That is, the designation "inertial" is applied not to worlds (as in Dowty 1979, where certain worlds are taken to be inertial relative to a time and world) but to observations, formalized below as fluents which are then strung together and collected in regular languages.

${ }^{2}$ That is, an event is conceptualized as a sequence of snapshots, as in Tenny 1987 - a conceptualization vigorously rejected in Jackendoff 1996. In defense, I work not just with strings but with languages (that I take some pains to check are regular). In section 6 , we shall see how the continuity that Jackendoff values can arise as the limit of finitary approximations from these languages. Short of that limit, issues of granularity and vagueness in natural language arguably call for the use of such approximations.

I have insisted on regular languages because in the present context, less is more: not only is computational complexity kept (by some measure) modest, but the finite-state machines constitute Kripke models for modal languages worth investigating. 
The horizontal line drawn in Figure 1 is presumably continuous, whereas our strings are discrete. But we can collect strings in sets that fill in as many of the points in that line as we would ever care to fill. And we can abstract over the fluents $f(x)$ to form one, $f_{\uparrow}$, capturing incremental change - although admittedly the modifier Previously leads to fluents that go beyond snapshots of single time points. Be that as it may, who is to say that the usual discrete picture of computation proceeding step-by-step is any less compelling than the illusion of continuity that motion pictures (if not comic strips) engender?

${ }^{14}$ In this case, the use of Previously may unnecessarily invite objections that our fluents record more than single snapshots.

${ }^{15}$ Replacing $\varphi$ in the language $\psi{ }_{\varphi}^{*}\left[, R\right.$ by $0 \square^{*} 1$ yields (under superposition) a language that no finite state machine can accept. (Apply a pumping argument after intersecting with $\psi 0,0, R]^{*}$.) Regularity has some bite even in the present context.

\section{References}

David Dowty. Word Meaning and Montague Grammar. Reidel, Dordrecht, 1979.

David Dowty. The effects of aspectual class on the temporal structure of discourse: semantics or pragmatics? Linguistics and Philosophy 9: 37-61, 1986.

Tim Fernando. Reichenbach's E, R and S in a finite-state setting. Sinn und Bedeutung 8, Frankfurt, 2003.

Tim Fernando. A finite-state approach to events in natural language semantics. Journal of Logic and Computation 14(1):79-92, 2004.

F. Hamm and M. van Lambalgen. Event Calculus, Nominalisation, and the Progressive. Linguistics and Philosophy 26(4):381-458, 2003.

Ray Jackendoff. The proper treatment of measuring out, telicity, and perhaps even quantification in English. Natural Language and Linguistic Theory 14:305-354, 1996.

H. Kamp and U. Reyle. From Discourse to Logic. Kluwer, Dordrecht, 1993.

J. McCarthy and P. Hayes. Some philosophical problems from the standpoint of artificial intelligence. In M. Meltzer and D. Michie (eds.), Machine Intelligence 4:463-502. Edinburgh University Press, 1969.

M. Moens and M. Steedman. Temporal ontology and temporal reference. Computational Linguistics 14(2):15-28, 1988.

Mark Steedman. The Productions of Time. Draft, ftp://ftp.cogsci.ed. ac.uk/pub/steedman/temporality/temporality.ps.gz, July 2000.

Carol Tenny. Grammaticalizing Aspect and Affectedness. PhD dissertation, Department of Linguistics and Philosophy, MIT, Cambridge, MA, 1987. 